\title{
A PAN-SHARPENING METHOD BASED ON GUIDED IMAGE FILTERING: A CASE STUDY OVER GF-2 IMAGERY
}

\author{
Yalan Zheng a, Mengxiao Guo a , Qinling Dai ${ }^{\text {b, c }}$, Leiguang Wang a, * \\ ${ }^{a}$ School of Forestry, Southwest Forestry University, Kunming, China, 650224 \\ ${ }^{b}$ School of Printing and Packaging, Wuhan University, Wuhan, China, 430079 \\ ${ }^{c}$ School of Material Engineering, Southwest Forestry University, Kunming, China, 650224
}

KEY WORDS: Remote Sensing, Image Fusion, Pan-sharpening, Guided Filter, GF-2 Imagery.

\begin{abstract}
:
The GaoFen-2 satellite (GF-2) is a self-developed civil optical remote sensing satellite of China, which is also the first satellite with the resolution of being superior to 1 meter in China. In this paper, we propose a pan-sharpening method based on guided image filtering, apply it to the GF-2 images and compare the performance to state-of-the-art methods. Firstly, a simulated low-resolution panchromatic band is yielded; thereafter, the resampled multispectral image is taken as the guidance image to filter the simulated low resolution panchromatic Pan image, and extracting the spatial information from the original Pan image; finally, the pan-sharpened result is synthesized by injecting the spatial details into each band of the resampled MS image according to proper weights. Three groups of GF-2 images acquired from water body, urban and cropland areas have been selected for assessments. Four evaluation metrics are employed for quantitative assessment. The experimental results show that, for GF-2 imagery acquired over different scenes, the proposed method can not only achieve high spectral fidelity, but also enhance the spatial details.
\end{abstract}

\section{INTRODUCTION}

With the rapid development of remote sensors, a great deal of optical earth observation satellites and digital aerial cameras can simultaneously obtain high spectral resolution multispectral (MS) and high spatial resolution panchromatic (Pan) images (Yun, 2012). However, due to the physical constraints, the spectral information is only rich in MS images, and it is difficult to acquire the images with both high spatial and spectral resolution. The images obtained from a single sensor often cannot meet applications, such as visual interpretation, change detection and detailed land cover classification, etc. Therefore, it is increasingly important to combine the strengths of the MS and Pan images (Dong, 2009) and (Ehlers, 2010).

To date, a large number of pan-sharpening methods have been proposed. Among them, component substitution (CS) (Qizhi, 2014) methods are more practical and widely used because of its fast speed in calculation and convenient implementation. The representative CS methods include principal component analysis (PCA), Gram-Schmidt transformation (GS), Intensity-HueSaturation (IHS) and University of New Brunswick (UNB) method (Zhang, 2004), etc. However, with more and more sensors with different spectral and spatial properties were launched, these existing methods show various limitations, and have not fully assessed on data sets captured by the new sensors (Zhang, 2004).

GF-2 satellite was launched on August, 2014. It is a selfdeveloped civilian optical remote sensing satellite of China, which is also the first satellite with the resolution of being superior to 1 meter in China. It can achieve a spatial resolution of

\footnotetext{
* Corresponding author: Leiguang Wang; Email:wlgbain@126.com
} 
0.8 meter with a swath of 48 kilometers in panchromatic mode, in contrast, 3.2 meter and 4 spectral bands in multispectral mode.

In this context, a pan-sharpening method based on guided image filtering is proposed and applies to GF-2 images. Experimental results show that the proposed method can achieve a better effectiveness on spectral information preservation and spatial detail enhancement.

\section{PROPOSED PAN-SHARPENING METHOD}

\subsection{Guided Image Filtering}

The guided image filtering is firstly proposed by $\mathrm{He}$ et al. (He, 2013 ) in 2010. It is quite popular due to its edge-preserving property and is widely used for combining features from two different source images, such as image matting/feathering (Levin, 2006), flash/no-flash de-noising (Petschnigg, 2004), HDR compression (Durand, 2002) and haze removal (He, 2011), etc. By using the guidance image to affect the process of filtering, the original image can be smoothed, meanwhile, the gradient information of the guidance image can also be retained.

The guided image filter (He, 2013) assumes that the filtering output is a local linear model between the filter output $Q$ and the guidance image $I$ in a local window $\omega_{k}$ centered at pixel $k$

$$
Q_{i}=a_{k} I_{i}+b_{k}, \forall i \in \omega_{k}
$$

where $a_{k}$ and $b_{k}$ are the linear coefficients considered to be constant in a small square image window $\omega_{k}$ of a radius $(2 r+1) \times(2 r+1)$. The local linear model guarantees $\nabla Q=a \nabla I$, that is, the filter output $Q$ has an edge only if the guidance image $I$ has an edge. Here, the coefficients $a_{k}$ and $b_{k}$ are computed by minimizing the following cost function:

$$
E\left(a_{k}, b_{k}\right)=\sum_{i \in \omega_{k}}\left[\left(a_{k} I_{i}+b_{k}-p_{i}\right)^{2}+\varepsilon a_{k}^{2}\right]
$$

where $\varepsilon$ is a regularization parameter that set up by users and prevents $a_{k}$ from being too large. The linear coefficients are directly resolved by the linear ridge regression (Draper, 1981) as follows:

$$
\begin{aligned}
& a_{k}=\frac{\frac{1}{|\omega|} \sum_{i_{\epsilon} \omega_{k}} I_{i} p_{i}-\mu_{k} \bar{p}_{k}}{\sigma_{k}^{2}+\varepsilon} \\
& b_{k}=\bar{p}_{k}-a_{k} \mu_{k} \\
& \bar{p}_{k}=\frac{1}{|\omega|} \sum_{i \epsilon \omega_{k}} p_{i}
\end{aligned}
$$

where $\mu_{k}$ and $\sigma_{k}^{2}$ are the mean and variance of $I \operatorname{in} \omega_{k},|\omega|$ is the number of pixels in $\omega_{k}$, and $\bar{p}_{k}$ is the mean of $p$ in $\omega_{k}$. However, all windows that contains $i$ will involve the pixel $i$, so different windows will have different values of $Q_{i}$. Then one effective method to resolve this problem is to average all the possible values of $Q_{i}$ to obtain the filtering output image $Q$.

Therefore, after calculating $\left(a_{k}, b_{k}\right)$ for all windows $\omega_{k}$ in the image, the filter result is computed by:

$$
\begin{aligned}
Q_{i} & =\frac{1}{|\omega|} \sum_{k: i \in \omega_{k}}\left(a_{k} I_{i}+b_{k}\right) \\
& =\bar{a}_{i} I_{i}+\bar{b}_{i}
\end{aligned}
$$

where $\bar{a}_{i}=\frac{1}{|\omega|} \sum_{k \in \omega_{i}} a_{k}$ and $\bar{b}_{i}=\frac{1}{|\omega|} \sum_{k \in \omega_{i}} b_{k}$.

\subsection{Proposed Pan-Sharpening Method}

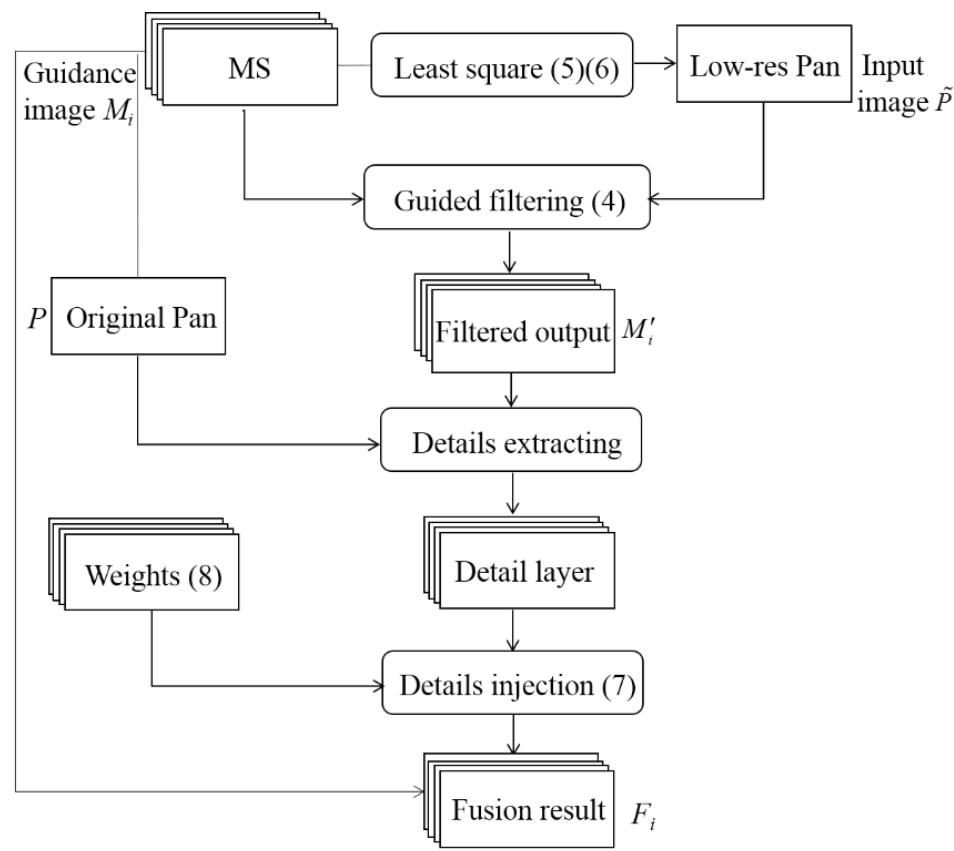

Figure 1. The processing flowchart of the proposed pansharpening method. 
As shown in Figure 1, this proposed pan-sharpening method consists of the following four procedures:

(1) The original multispectral image is registered and resampled as the same size as the Pan image $P$.

(2) By minimizing the residual sum of squares (5), the weights $w_{i}$ (with $\left.i=1,2,3,4\right)$ can be easily estimated.

$$
R S S\left(w_{i}\right)=\sum_{x} \sum_{y}\left(P(x, y)-\sum_{i=1}^{4} w_{i} M_{i}(x, y)\right)^{2}
$$

Thereafter, by introducing (5) into (6), a synthetic lowresolution panchromatic image $\tilde{P}$ can be obtained.

$$
\tilde{P}=\sum_{i=1}^{4} w_{i} M_{i}
$$

where $\tilde{P}$ is the simulated low resolution panchromatic image and $w_{i}$ is the weight for the $i$-th band $M_{i}(x, y)$, which is constant for the given band.

(3) Take each $M_{i}$ (with $i=1,2,3,4$ ) as the guidance image to guide the filtering process of low resolution Pan image $\tilde{P}$, and then obtain the filter output $M_{i}^{\prime}$ (with $i=1,2,3,4$ ) respectively.

(4) The pan-sharpening result $F_{i}$ is obtained by extracting the spatial information of the Pan image, and injecting into the resampled MS image $M_{i}$ according to weight $\alpha_{i}(x, y)$, its definition is as follows:

$$
\begin{gathered}
F_{i}(x, y)=\left(P(x, y)-M_{i}^{\prime}(x, y)\right) \times \alpha_{i}(x, y)+M_{i}(x, y), i \in n \\
\alpha_{i}(x, y)=\frac{1}{\sqrt{\sum_{(x, y) \in w_{(x, y)}}\left(M_{i}(x, y)-P(x, y)\right)^{2}}}, i \in n
\end{gathered}
$$

where $F_{i}(x, y)$ is the fusion image, $P(x, y)$ is original Pan image, $M_{i}(x, y)$ is the resampled MS image, $M_{i}^{\prime}(x, y)$ is the filtering output, $\alpha_{i}(x, y)$ is the weight corresponding to $i$-th MS band at the position $(x, y), w_{(x, y)}$ expresses a local square window centered at $(x, y),(p, q)$ denotes a pixel in the local square window, $i$ is the band number of MS image and $n=\{1,2,3,4\}$ is the total band number of the MS image. It is obvious that the greater the distance, the smaller the weight; otherwise, the weight should be large.

\section{EXPERIMENTAL RESULTS AND COMPARISONS}

\subsection{Experimental Setting}

For analysis and comparison of the proposed and other pansharpening methods, three pairs of Gaofen-2 imagery were selected for test in this paper. Table 1 shows the characteristics of this dataset. The test images were acquired over Guangzhou, China, three scenes including urban, water body and the cropland areas. The MS image consists of four bands and the spectral range of the MS bands is exactly covered by the range of the Pan band. The size of each image is $1000 \times 1000$.

\begin{tabular}{ll}
\hline Spatial resolution & MS:3.2m \\
& Pan:0.8m \\
\hline & Blue:450-520nm \\
Green:520-590nm & Red:630-690nm \\
& NIR:770-890nm \\
Spectral range & Pan:450-900nm \\
& Guangzhou \\
Locations of images & Urban, water body, \\
cropland, etc. \\
Land cover types & MS: $250 \times 250$ \\
Image size & Pan: $1000 \times 1000$ \\
\hline
\end{tabular}

Table 1. Characteristics of the employed GF-2 datasets

In order to verify the effectiveness of the proposed approach, three state-of-the-art fusion methods, including the GS transformation (Laben, 2000) and NND method (Sun, 2014) in ENVI software, and UNB method (Zhang, 2004) in PCI Geomatica software, were employed in the experiments for comparison.

\subsection{Assessment Metrics}

Four widely used metrics are selected for quantitative assessment, they are the entropy, the correlation coefficient (CC) (Klonus, 2007), the universal image quality index (UIQI) (Wang, 2002) and the relative dimensionless global error in synthesis (ERGAS) (Ranchin, 2000). The resampled MS image is taken as the reference image.

1) The entropy can be used to measure how many spatial information that the fused image contains. The higher the entropy is, the better the quality of the fused image will be. Its 
definition is as follows:

$$
\text { Entropy }=-\sum_{0}^{255} F(i) \log _{2} F(i)
$$

where Entropy is the entropy of image, and $F(i)$ is the probability of pixel value $i$ in the image.

2) CC (Klonus, 2007) measures the correlation between the MS image and the fused image. The higher correlation value indicates a better correspondence between the MS image and the fused image. It is expressed as:

$$
C C=\frac{\sum_{i=1}^{m} \sum_{j=1}^{n}[M(i, j)-\bar{M}][F(i, j)-\bar{F}]}{\sqrt{\left\{\left[\sum_{i=1}^{m} \sum_{j=1}^{n} M(i, j)-\bar{M}\right]^{2}\right\}\left\{\left[\sum_{i=1}^{m} \sum_{j=1}^{n} F(i, j)-\bar{F}\right]^{2}\right\}}}
$$

$\bar{M}$ and $\bar{F}$ stand for the mean values of the original MS and fused image, and CC is calculated globally for the entire image.

3) UIQI (Wang, 2002) models any distortion as a combination of three different factors: loss of correlation, luminance distortion and contrast distortion. Its definition is given by:

$$
U I Q I=\frac{\sigma_{x y}}{\sigma_{x} \sigma_{y}} \times \frac{2 \overline{x y}}{(\bar{x})^{2}+(\bar{y})^{2}} \times \frac{2 \sigma_{x} \sigma_{y}}{\sigma_{x}^{2}+\sigma_{y}{ }^{2}}
$$

where $\bar{x}$ and $\bar{y}$ are the mean values of the fused and original images respectively, $\sigma_{x}$ and $\sigma_{y}$ are the standard deviation of the fused and original images respectively.

4) ERGAS (Ranchin, 2000) evaluates the overall spectral distortion of the pan-sharpened image. It is defined as:

$$
E R G A S \stackrel{\Delta}{=} 100 \frac{d_{P}}{d_{M S}} \sqrt{\frac{1}{K} \sum_{i=1}^{K} \frac{\operatorname{RMSE}^{2}(i)}{M E A N^{2}(i)}}
$$

where $d_{P} / d_{M S}$ is the ratio between pixel sizes of the Pan and MS images, $K$ is the number of bands, $\operatorname{MEAN}(i)$ is the mean of the $i$-th band, whereas $\operatorname{RMSE}(i)$ is the root-mean-square error between the $i$-th band of the reference image and the $i$-th band of the fused image.

\subsection{Results and Performance Comparison}

As shown in Fig. 2 to Fig. 4, local patches with size of $400 \times 600$ pixels over different land cover types are clipped from the fused results and displayed in true color with the same stretching mode. Table 2 to Table 4 correspond to these quantitative evaluation results. The best performance of each metric is in bold.
After the visual comparison, the fused images yielded by the NND method have obvious spectral distortion on the green vegetation regions, especially the Figure 3 and Figure 4, not match the original deep green color. This may be due to the NND method is more suitable for fusing low resolution images, like Landsat 7 data, etc. While UNB and GS methods achieve excellent performance on spatial quality. Moreover, as it can be seen from all these figures, the proposed method has better effectiveness especially on spectral fidelity.

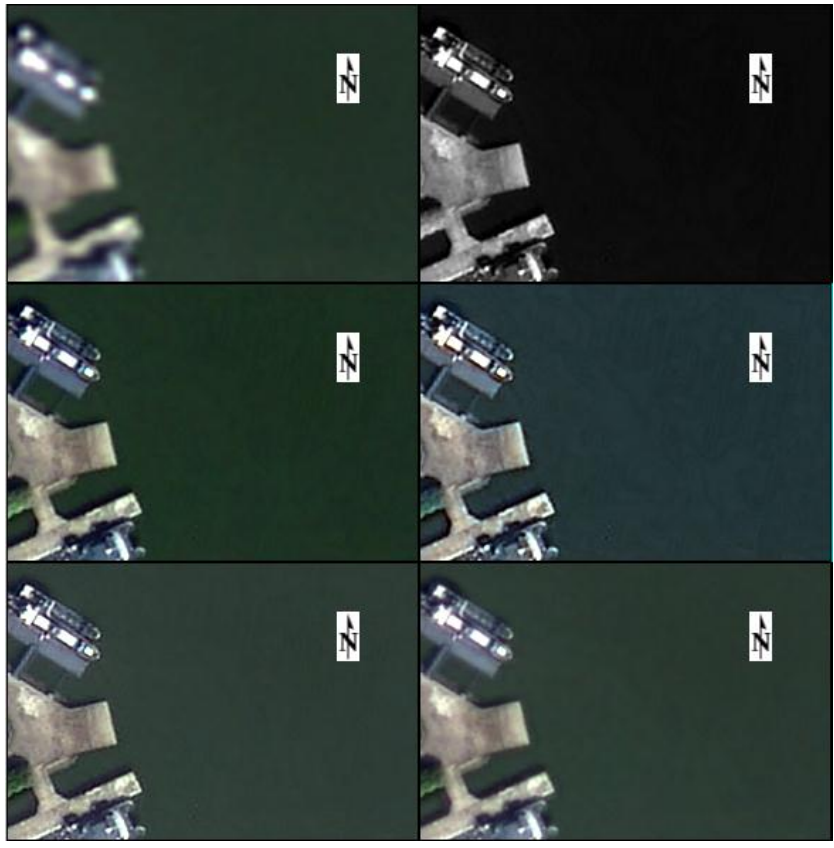

Figure 2. The fused images with different methods of GF-2 image over the water body. From left to right, up and down:

MS; PAN; GS; NND; UNB and the proposed method.

\begin{tabular}{ccccc}
\hline Method & Entropy & UIQI & CC & ERGAS \\
\hline MS & 5.852 & & & \\
GS & 5.622 & 0.834 & 0.885 & 34.042 \\
NND & 5.402 & 0.714 & 0.862 & 41.577 \\
UNB & 5.266 & 0.871 & 0.914 & 21.203 \\
Proposed & $\mathbf{5 . 6 2 5}$ & $\mathbf{0 . 9 3 2}$ & $\mathbf{0 . 9 5 9}$ & $\mathbf{2 0 . 7 7 9}$ \\
\hline
\end{tabular}

Table 2. Quality evaluation of fused images: Water body (corresponding to Figure 2). The best performance of each metric is in bold.

For quantitative assessments, all of the metric scores of the proposed method are the best in Table 2 and Table 4. In Table 3, 
the ERGAS value of the proposed method is the second best, but other metrics are all the best. This demonstrates that the proposed method achieves better performance on spectral preserving and spatial enhancement than other methods. It can attribute to the edge-preserving feature of the guided filtering, on the other hand, it is also because the proposed method takes advantage of the proper weights to inject the spatial details into each band of the resampled MS image. Furthermore, the results of quality assessment also agree with the visual comparison.

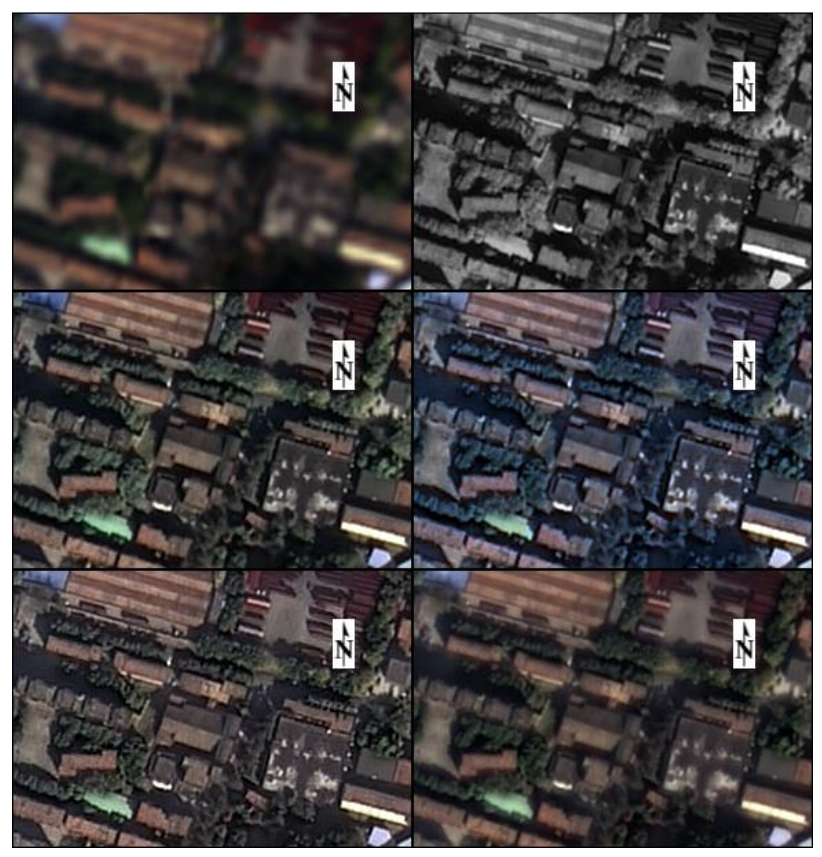

Figure 3. The fused images with different methods of GF-2 image over the urban. From left to right, up and down: MS;

PAN; GS; NND; UNB and the proposed method.

\begin{tabular}{ccccc}
\hline Method & Entropy & UIQI & CC & ERGAS \\
\hline MS & 6.395 & & & \\
GS & 6.187 & 0.843 & 0.874 & $\mathbf{2 0 . 5 4 2}$ \\
NND & 6.060 & 0.689 & 0.861 & 42.647 \\
UNB & 5.816 & 0.785 & 0.866 & 25.974 \\
Proposed & $\mathbf{6 . 3 1 5}$ & $\mathbf{0 . 9 3 6}$ & $\mathbf{0 . 9 5 4}$ & 21.262 \\
\hline
\end{tabular}

Table 3. Quality evaluation of fused images: Urban

(corresponding to Figure 3). The best performance of each metric is in bold.

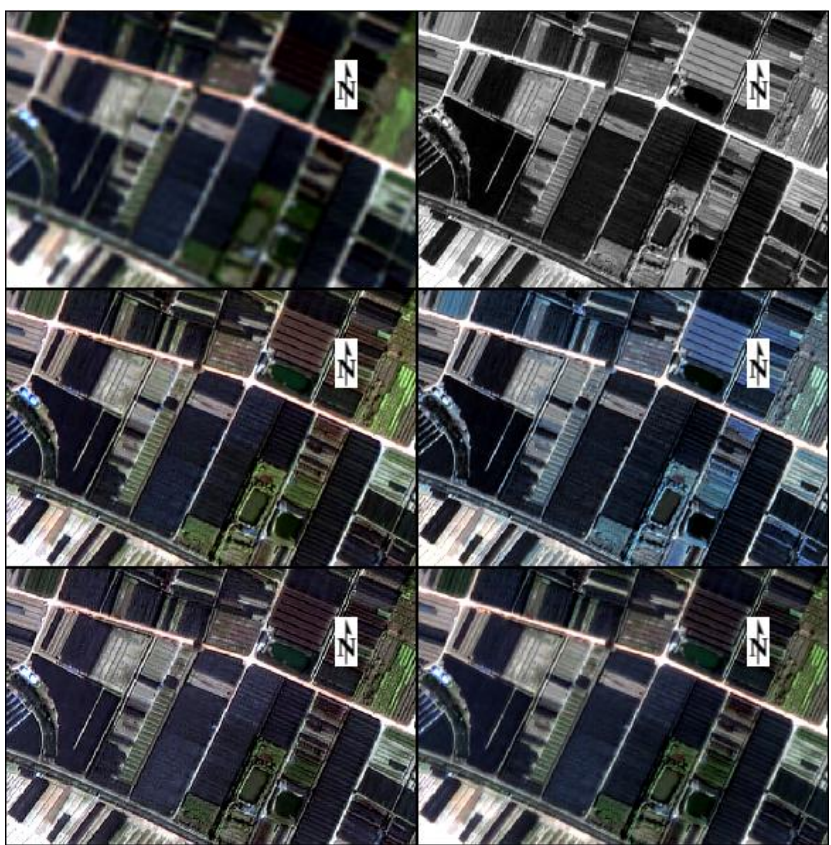

Figure 4 . The fused images with different methods of GF-2 image over the cropland. From left to right, up and down: MS; PAN; GS; NND; UNB and the proposed method.

\begin{tabular}{ccccc}
\hline Method & Entropy & UIQI & CC & ERGAS \\
\hline MS & 6.865 & & & \\
GS & 5.971 & 0.798 & 0.890 & 26.401 \\
NND & 6.028 & 0.676 & 0.807 & 66.224 \\
UNB & 5.443 & 0.631 & 0.879 & 40.354 \\
Proposed & $\mathbf{6 . 1 3 1}$ & $\mathbf{0 . 8 8 7}$ & $\mathbf{0 . 9 4 3}$ & $\mathbf{1 8 . 6 6 9}$ \\
\hline
\end{tabular}

Table 4. Quality evaluation of fused images: Cropland (corresponding to Figure 4). The best performance of each metric is in bold.

\section{CONCLUSION}

In this research, a pan-sharpening method based on guided image filtering is proposed, and applying it to GF-2 images. A great number of experimental results and quality assessments have demonstrated that, for GF-2 imagery acquired over different scenes, the proposed method can consistently achieve high spectral fidelity and enhance the spatial details independent of the content of the images. Furthermore, how to adaptively select the window size of weight calculation and estimate the parameters of guided filtering will be future researches. 


\section{ACKNOWLEDGEMENT}

This project is supported by national Natural Science Foundation of China under grants of 41571372 and 41301470.

\section{REFERENCES}

Dong J, Zhuang D, Huang Y, Fu J. Advances in Multi-Sensor Data Fusion: Algorithms and Applications[J]. Sensors. 2009,9(10):7771-84.

Draper, N., Smith, H. Applied Regression Analysis. 2 edn. John Wiley (1981)

Durand, F., Dorsey, J. Fast bilateral filtering for the display of high-dynamic-range images. SIGGRAPH (2002)

Ehlers M, Klonus S, Johan Åstrand P, Rosso P. Multi-sensor image fusion for pansharpening in remote sensing[J]. International Journal of Image and Data Fusion. 2010,1(1):2545 .

He K, Sun J, Tang X. Guided Image Filtering [J]. IEEE Transactions on Pattern Analysis and Machine Intelligence. 2013,35 (6):1397-409.

He K, Sun J, Tang X. Single Image Haze Removal Using Dark Channel Prior[J]. IEEE Transactions on Pattern Analysis and Machine Intelligence. 2011,33(12):2341-53.

Klonus, S. and Ehlers, M. Image fusion using the Ehlers spectral characteristics preserving algorithm. GIScience and Remote Sensing, 2007, 44 (2): 93-116.

Laben CA, Brower BV. Process for enhancing the spatial resolution of multispectral imagery using pan-sharpening. Google Patents; 2000.

Levin, A., Lischinski, D., Weiss, Y. A closed form solution to natural image matting. CVPR (2006)

Petschnigg, G., Agrawala, M., Hoppe, H., Szeliski, R., Cohen, M., Toyama, K. Digital photography with flash and no-flash image pairs. SIGGRAPH (2004)

Qizhi X, Bo L, Yun Z, Lin D. High-Fidelity Component Substitution Pansharpening by the Fitting of Substitution Data[J]. Geoscience and Remote Sensing, IEEE Transactions on. 2014,52(11):7380-92.

Ranchin T, Wald L. Fusion of high spatial and spectral resolution images_The ARSIS concept and its implementation[J]. Photogrammetric Engineering \& Remote Sensing. 2000,66(1):49-61.

Sun W, Chen B, Messinger DW. Nearest-neighbor diffusionbased pan-sharpening algorithm for spectral images [J]. Optical Engineering. 2014, 53(1):013107.

Wang Z, Bovik AC. A universal image quality index[J]. IEEE Signal Processing Letters. 2002,9(3):81-4.

Yun Z, Mishra RK, editors. A review and comparison of commercially available pan-sharpening techniques for high resolution satellite image fusion. Geoscience and Remote Sensing Symposium (IGARSS), 2012 IEEE International; 2012 22-27 July 2012.

Zhang Y. Understanding image fusion[J]. Photogrammetric engineering and remote sensing. 2004,70(6):657-61. 